BULLETIN OF THE

AMERICAN MATHEMATICAL SOCIETY

Volume 80, Number 6, November 1974

\title{
WITT CLASSES OF INTEGRAL REPRESENTATIONS OF AN ABELIAN $p$-GROUP
}

BY J. P. ALEXANDER, P. E. CONNER, G. C. HAMRICK AND J. W. VICK ${ }^{1}$

Communicated by Glen Bredon, April 19, 1974

1. Introduction. For a Dedekind domain, $R$, the orthogonal and symplectic representations of a finite group, $\pi$, on finitely-generated projective inner-product modules over $R$ admit a Witt equivalence relation, and the resulting equivalence classes form a commutative algebra, $W_{*}(R, \pi)$, over the Witt ring of $R$. This concept has received considerable attention recently $[2],[3],[4]$. Our interest is motivated by the fact that $\omega_{*}(Z, \pi)$ is so very specifically related to the bordism classification of smooth, orientation preserving actions of $\pi$ on closed even-dimensional manifolds. We shall discuss

(1.1) TheOREM. If, for $p$ an odd prime, $\pi$ is an abelian p-group then $W_{*}(Z, \pi)$ contains no torsion.

A corollary of (1.1) is that for an action $\left(\pi, M^{2 k}\right)$ of such a group on a closed oriented manifold, the Atiyah-Singer-Segal $G$-signature theorem [1] determines the integral Witt class of $\left(\pi, H^{*}(M ; Z) /\right.$ tor $)$ uniquely. The present techniques may also be applied to determine $W_{*}(Z, \pi)$ for an abelian 2-group, however torsion is present always. Thus for an orientation preserving action $\left(\pi, M^{2 k}\right)$ of an abelian 2-group, a torsion valued invariant, as well as the multisignature, must be computed.

By rough analogy with $[5, I V,(3.3)]$ there is

(1.2) LemmA. For any p-group

$$
W_{2}(Z, \pi) \simeq W_{2}(Z(1 / p), \pi)
$$

and there is a split short exact sequence

$$
0 \rightarrow \omega_{0}(Z, \pi) \rightarrow W_{0}(Z(1 / p), \pi) \rightarrow \omega\left(Z_{p}\right) \rightarrow 0 .
$$

AMS (MOS) subject classifications (1970). Primary 57D85; Secondary $10 \mathrm{C05}$.

Key words and phrases. Bordism, representation, Witt ring.

1 The authors were supported in part by the National Science Foundation. 
We use the subscripts 0 and 2 respectively to denote orthogonal and symplectic representations.

2. Cyclic $p$-groups. From this point we restrict our attention to odd primes. For $n \geqslant 0$ we denote by $Q(\lambda)$ the $p^{n+1}$-cyclotomic extension of the rationals. In the ring of algebraic integers, $Z(\lambda)$, there is the multiplicative subset, $S$, generated by the rational prime, $p$, and $S^{-1} Z(\lambda)=D \subset$ $Q(\lambda)$ is a Dedekind domain invariant under complex conjugation. We may thus speak of the Witt ring of Hermitian inner-product modules over $D$, $H_{0}(D)$, and by introducing skew-Hermitian inner-products there is $\mathrm{H}_{2}(D)$ and hence an algebra, $H_{*}(D)$.

(2.1) LEMMA. For $n \geqslant 0$ there is an additive isomorphism

$$
W_{*}\left(Z(1 / p), Z_{p^{n+1}}\right) \simeq W_{*}\left(Z(1 / p), Z_{p^{n}}\right) \oplus H_{*}(D) .
$$

Very briefly, we consider a $\left(Z_{p^{n+1}}, V\right)$ where $V$ is an inner-product module over $Z(1 / p)$ and choose a generator $T \in Z_{p^{n+1}}$. With $\tau=T^{p^{n}}$,
we introduce into $V$ a selfadjoint projection operator

$$
\sum v=\left(v+\tau(v)+\cdots+\tau^{p-1}(v)\right) / p .
$$

This yields an orthogonal decomposition $V=I \oplus I^{\perp}$ into the image, $I$, of $\Sigma$ and the kernel, $I^{\perp}$. On $I$ the subgroup generated by $\tau$ acts trivially, so we may replace $Z_{p^{n+1}}$ by the quotient group $Z_{p^{n}}$. Now $D$ is the quotient of the group ring $Z(1 / p)\left(Z_{p^{n+1}}\right)$ by the principal ideal which $1+\tau+\cdots+\tau^{p-1}$ generates. In this fashion $I^{\perp}$ becomes a projective $D$-module. The (skew-)Hermitian inner-product on $I^{\perp}$ is $[v, w]=$ $\Sigma_{j}\left(v, T^{j} w\right) \lambda^{j}$

At this point standard algebraic number theory intervenes in proving

(2.2) Lemma. The Hermitian Witt ring $H_{0}(D)$ has no torsion.

Denoting by $Q\left(\lambda+\lambda^{-1}\right)$ the subfield of real elements in $Q(\lambda)$, we may paraphrase the discussion in [5, IV, §4] to show that $v \in Q\left(\lambda+\lambda^{-1}\right)^{*}$ can, up to mulitplication by a Hermitian square, be realized as the discriminant of a Hermitian inner-product module over $D$ with even rank if and only if $L L^{-}=v D$ for some fractional $D$-ideal $L \subset Q(\lambda)$. The key lemma then is

(2.3) LEMmA. If $L L^{-}=v D$ then $v$ is a Hermitian square if and only if it is positive in every ordering of $Q\left(\lambda+\lambda^{-1}\right)$. 
The lemma depends on the fact that only the rational prime $p$ ramifies in $Q(\lambda)$. It then proceeds from a combination of the local norm index theorem for units [6, IX, p. 187] with the reciprocity law for Hilbert symbols in the number field $Q\left(\lambda+\lambda^{-1}\right)$. As a consequence of Landherr [5, p. 118, Example 4], this lemma eliminates torsion in $H_{0}(D)$. It is possible to determine $H_{0}(D)$ completely. It is necessary to produce elements $v \in Q\left(\lambda+\lambda^{-1}\right)^{*}$ with arbitrarily prescribed signs and satisfying $L L^{-1}=v D$. This cannot be accomplished, in general, by only selecting units in $D^{*} \cap Q\left(\lambda+\lambda^{-1}\right)^{*}$, and the argument involves a careful analysis of the role of the homology groups of $Z_{2}$ acting on the ideal class group of $D$ via conjugation of fractional $D$-ideals.

Beginning with $n=0$, Lemmas (2.2), (2.1) and (1.2) are combined inductively to yield (1.1) for the cyclic $p$-groups. Since $D^{*}$ contains an imaginary unit, $H_{0}(D) \simeq H_{2}(D)$ additively so there is no special problem in handling the symplectic case.

3. The general case. We express $\pi$ as a direct sum $\pi=\pi_{1} \oplus Z_{p^{n+1}}$ with $n$ as large as possible. Now proceeding as in (2.1) we split $W_{*}(Z(1 / p), \pi)$ into a direct sum $W_{*}\left(Z(1 / p), \pi_{1} \oplus Z_{p^{n}}\right) \oplus H_{*}\left(D, \pi_{1}\right)$. From our choice of $n$ we may identify the character group $\pi_{1}^{*}$ with $\operatorname{Hom}\left(\pi_{1}, D^{*}\right)$. It follows readily that $H_{*}\left(D, \pi_{1}\right) \simeq Z\left(\pi_{1}^{*}\right) \otimes H_{*}(D)$. Combining (2.1) and (1.2) with this observation, (1.1) is established.

For a specific example if $p$ is odd, the multisignature is an isomorphism of $W_{*}\left(Z, Z_{p}\right)$ onto the subring of the group ring $Z\left(Z_{p}\right)$ consisting of those elements which can be expressed in the form

$$
m_{0} \cdot e+\sum_{1}^{k} m_{j}\left(\tau^{j}+\tau^{-j}\right)+\sum_{1}^{k} n_{j}\left(\tau^{j}-\tau^{-j}\right),
$$

where $k=(p-1) / 2$ and the integral coefficients satisfy $m_{1}=m_{2}=\cdots=$ $m_{k}(\bmod 2)$ and $n_{1}=n_{2}=\cdots=n_{k}(\bmod 2)$.

ADDED IN PROOF. Theorem (1.1) can be proven for general $p$-groups $\pi$, for example by the induction techniques of Dress [2].

\section{REFERENCES}

1. M. F. Atiy ah and I. M. Singer, The index of elliptic operators. III, Ann. of Math (2) 87 (1968), 546-604. MR 38 \#245.

2. A. Dress, Induction and structure theorems for Grothendieck and Witt rings of orthogonal representations of finite groups, Bull. Amer. Math. Soc. 79 (1973), 741-745. 
1182 J. P. ALEXANDER, P. E. CONNER, G. C. HAMRICK AND J. W. VICK

3. A. Frölich and A. M. McEvett, The representation of groups by automorphisms of forms, J. Algebra 12 (1969), 114-133. MR 39 \#1569.

4. A. Frölich, Orthogonal and symplectic representations of groups, Proc. London Math. Soc. (3) 24 (1972), 470-506. MR 46 \#7362.

5. J. W. Milnor and D. Husemoller, Symmetric bilinear forms, Ergebnisse der Mathematik und ihrer Grenzgebiete, Band 73, Springer-Verlag, Berlin and New York, 1973.

6. S. Lang, Algebraic number theory, Addison-Wesley, Reading, Mass., 1970. MR 44 \#181.

DEPARTMENT OF MATHEMATICS, LOUISIANA STATE UNIVERSITY, BATON ROUGE, LOUISIANA 70803

DEPARTMENT OF MATHEMATICS, UNIVERSITY OF TEXAS AT AUSTIN, AUSTIN, TEXAS $\mathbf{7 8 7 1 2}$ 